\title{
MRI Planimetry and Magnetic Resonance Parkinsonism Index in the Differential Diagnosis of Patients with Parkinsonism
}

\author{
(D) V.C. Constantinides, (D).P. Paraskevas, (D). Velonakis, DP. Toulas, DE. Stamboulis, and (DE. Kapaki
}

\begin{abstract}
BACKGROUND AND PURPOSE: Differential diagnosis of multiple system atrophy, progressive supranuclear palsy, and corticobasal degeneration from Parkinson disease on clinical grounds is often difficult. MR imaging biomarkers could assist in a more accurate diagnosis. We examined the utility of MR imaging surface measurements (MR imaging planimetry) in the differential diagnosis of patients with parkinsonism.
\end{abstract}

MATERIALS AND METHODS: Fifty-two patients with Parkinson-plus (progressive supranuclear palsy, $n=24$; corticobasal degeneration, $n=9$; multiple system atrophy, $n=19$ ), 18 patients with Parkinson disease, and 15 healthy controls were included. Corpus callosum, midbrain, and pons surfaces; relevant indices; and the Magnetic Resonance Parkinsonism Index were calculated. Corpus callosum subsection analysis was performed, and the corpus callosum posteroanterior gradient was introduced.

RESULTS: A Magnetic Resonance Parkinsonism Index value of $>12.6$ discriminated progressive supranuclear palsy from other causes of parkinsonism with a $91 \%$ sensitivity and $95 \%$ specificity. No planimetry measurement could accurately discriminate those with multiple system atrophy with parkinsonism from patients with Parkinson disease. A corpus callosum posteroanterior gradient value of $\leq 191$ was highly specific (97\%) and moderately sensitive (75\%) for the diagnosis of corticobasal degeneration versus all other groups. A midbrainto-corpus callosum posteroanterior gradient ratio of $\leq 0.45$ was highly indicative of progressive supranuclear palsy over corticobasal degeneration (sensitivity $86 \%$, specificity $88 \%$ ).

CONCLUSIONS: MR imaging planimetry measurements are potent imaging markers of progressive supranuclear palsy and promising markers of corticobasal degeneration but do not seem to assist in the diagnosis of multiple system atrophy with parkinsonism.

ABBREVIATIONS: $C B D=$ corticobasal degeneration; $C C=$ corpus callosum; $C C_{\mathrm{P}-\mathrm{A} \text { grad }}=$ corpus callosum posteroanterior gradient; $\mathrm{MRPI}=\mathrm{Magnetic}$ Resonance Parkinsonism Index; MSA = multiple system atrophy; MSA-C = multiple system atrophy cerebellar type; MSA-P = multiple system atrophy with parkinsonism; $\mathrm{PD}=$ Parkinson disease; PSP = progressive supranuclear palsy

M ultiple system atrophy (MSA), progressive supranuclear palsy (PSP), and corticobasal degeneration (CBD) are neurodegenerative parkinsonian disorders that compose the Parkinson-plus syndromes. Despite the presence of distinct clinical features, differential diagnosis is often difficult. ${ }^{1}$ Diagnostic criteria for PSP and MSA lack sensitivity, particularly in atypical cases and at the early stages of the diseases. ${ }^{2,3}$ Recently established criteria of CBD, on

Received November 9, 2017; accepted after revision February 7, 2018.

From the 1st Department of Neurology (V.C.C., G.P.P., E.S., E.K.), National and Kapodistrian University of Athens, Medical School, Eginition Hospital, Athens, Greece; and Research Unit of Radiology (G.V., P.T.), 2nd Department of Radiology, National and Kapodistrian University of Athens, Medical School, Athens, Greece.

Please address correspondence to Vasilios C. Constantinides, MD, 1st Department of Neurology, National and Kapodistrian University of Athens, Medical School, Eginition Hospital, 72-74 Vas. Sofias Ave, Athens, Greece, P.C. 11528; e-mail: vassilis.kon@hotmail.com, vconstan@med.uoa.gr

$\equiv$ Indicates article with supplemental on-line table.

Indicates article with supplemental on-line photos.

http://dx.doi.org/10.3174/ajnr.A5618 the other hand, are considered to lack specificity, and misdiagnosis may reach up to $50 \% .{ }^{4}$ Thus, objective diagnostic markers are needed to improve diagnostic accuracy in parkinsonian disorders.

PSP is characterized by relatively selective midbrain and superior cerebellar peduncle atrophy, ${ }^{5}$ as well as anterior corpus callosum thinning. ${ }^{6}$ Pons and middle cerebellar peduncle atrophy is pronounced in MSA. ${ }^{7}$ Patients with CBD present with asymmetric frontoparietal cortical atrophy as well as middle and posterior corpus callosum (CC) thinning. ${ }^{8,9}$ By means of MR imaging planimetry, the midbrain-to-pons surface ratio and the Magnetic Resonance Parkinsonism Index (MRPI), calculated by multiplying the pontine-to-midbrain area ratio by the middle cerebellar peduncle-to-superior cerebellar peduncle width ratio, have been suggested to assist in a more accurate and earlier diagnosis of PSP and, to a lesser extent, of MSA. ${ }^{10}$ To the best of our knowledge, no MR imaging planimetry study has incorporated midbrain, pons, and CC surface measurements in all 3 Parkinson-plus syndromes.

The aim of the present study was to examine the utility of 


\begin{tabular}{|c|c|c|c|c|c|c|}
\hline & $\begin{array}{c}\text { Controls } \\
n=15\end{array}$ & $\begin{array}{c}\text { PSP } \\
n=24\end{array}$ & $\begin{array}{l}\text { CBD } \\
n=9\end{array}$ & $\begin{array}{l}\text { MSA } \\
n=19\end{array}$ & $\begin{array}{c}\text { PD } \\
n=18\end{array}$ & $P$ Value \\
\hline \multicolumn{7}{|l|}{ Demographic data } \\
\hline $\operatorname{Sex}(M / F)$ & $8: 7$ & 13:11 & $4: 5$ & 14:5 & $10: 8$ & $.454^{\mathrm{b}}$ \\
\hline Age $(y r)$ & $62.6(9.0)$ & $63.2(6.8)$ & $67.9(5.6)$ & $64.2(7.1)$ & $64.4(9.3)$ & $.433^{\mathrm{c}}$ \\
\hline Disease duration (yr) & NA & $3.3(1.8)$ & $3.3(1.8)$ & $3.1(2.1)$ & $10.6(6.1)$ & $<.001^{\mathrm{C}}$ \\
\hline \multicolumn{7}{|l|}{ Clinical data } \\
\hline UPDRS III & NA & $21.3(9.8)$ & $25.2(7.1)$ & $19.6(19.1)$ & $32.3(11.5)$ & $.875^{c}$ \\
\hline UPDRS $_{\text {brad }}$ & NA & $6.1(4.7)$ & $12.5(3.6)$ & $7.7(9.3)$ & $14.5(8.4)$ & $.134^{c}$ \\
\hline UPDRS $_{\text {rig }}$ & NA & $4.1(3.0)$ & $7.2(4.4)$ & $3.9(5.6)$ & $9.2(6.5)$ & $.266^{\mathrm{c}}$ \\
\hline \multicolumn{7}{|c|}{ White matter lesion burden assessment } \\
\hline Fazekas PVH score & $0.38(0.65)$ & $0.71(0.75)$ & $0.70(0.48)$ & $0.37(0.50)$ & $0.47(0.62)$ & $.321^{c}$ \\
\hline Fazekas DWMH score & $0.77(0.73)$ & $0.54(0.66)$ & $0.40(0.52)$ & $0.53(061)$ & $0.53(0.51)$ & $.684^{c}$ \\
\hline
\end{tabular}

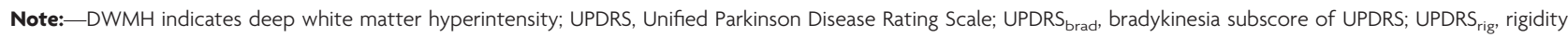
subscore of UPDRS; PVH, periventricular; NA, not applicable.

${ }^{a}$ Data are presented as mean (SD).

${ }^{b} \chi^{2}$ test.

c ANOVA.

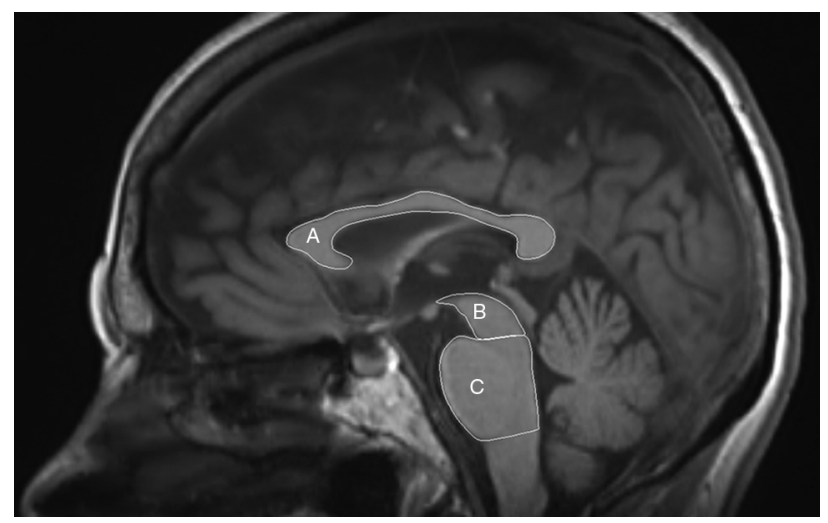

FIG 1. MR imaging planimetry measurements. Midsagittal T1weighted image depicts corpus callosum $(A)$, midbrain tegmentum (B), and pons $(C)$ surfaces.

already-suggested MR imaging brain stem surfaces and ratios in the differential diagnosis of patients with parkinsonism, including CBD, and to assess novel ones (incorporating the CC surface and CC subsections) in a well-characterized, prospectively diagnosed cohort.

\section{MATERIALS AND METHODS \\ Patients}

Patients were consecutively and prospectively recruited (between 2011 and 2014) as part of the Parkinson-Plus Registry of the 1st Department of Neurology, National and Kapodistrian University of Athens. Detailed neurologic history was obtained, and a comprehensive neurologic examination was performed in all patients, with a follow-up of at least 2 years. Standard laboratory tests to exclude secondary causes of parkinsonism were performed in all patients, as appropriate.

All patients fulfilled the established the diagnostic criteria for probable PSP with a Richardson syndrome phenotype, ${ }^{11}$ probable corticobasal degeneration with a probable corticobasal syndrome phenotype, ${ }^{4}$ or multiple system atrophy. ${ }^{12}$ None of the included patients had a history of stroke or other focal lesions, in accordance with the exclusion criteria of the established diagnostic criteria.

The Unified Parkinson's Disease Rating Scale III was applied in all patients to measure the severity of parkinsonism. The bradykinesia and rigidity subscores were also included.
CSF $\beta$ amyloid $\left(\mathrm{A} \beta_{42}\right), \tau$ protein $\left(\tau_{\mathrm{T}}\right)$, and phosphorylated $\tau$ protein at threonine-181 $\left(\tau_{\mathrm{P}-181}\right)$ were measured in all patients, as described elsewhere. ${ }^{13}$ Patients with a typical Alzheimer disease CSF biochemical profile were excluded (ie, decreased $\mathrm{A} \beta_{42}$, elevated $\tau_{\mathrm{T}}$ and $\tau_{\mathrm{P}-181}$ according to cutoff values of our laboratory). ${ }^{13}$ Thus 5 patients fulfilling the criteria for probable CBD were excluded.

Finally, 52 patients with Parkinson-plus were included (PSP, $n=24$; CBD, $n=9$; MSA, $n=19$ ). For comparison, a group of 18 patients with Parkinson disease (PD) diagnosed according to the UK Parkinson's Disease Society Brain Bank clinical diagnostic criteria were used. ${ }^{14}$ Furthermore, 15 otherwise healthy individuals, with no history of neurologic, psychiatric, or other major diseases and no signs of parkinsonism or cognitive dysfunction who were admitted to our department for nonrelevant issues (headache, dizziness, and so forth) served as a control group. Demographic data of our cohort are presented in Table 1.

\section{Ethical Issues}

All patients (or the next of kin caretaker in cases of compromised mental capacity) gave written informed consent for participation in the study, which was performed according to the ethics guidelines of the 1964 Declaration of Helsinki and had the approval of the Scientific and Ethics Committee of Eginition Hospital.

\section{MR Imaging Acquisition}

MR imaging was performed on a variety of high-field (1.5T to 3T) MR imaging units. The sequences included T1-weighted axial, sagittal, and coronal images or 3D T1-weighted turbo field echo sequences. MR imaging specifications were as follows: TR range, 500-650 ms; TE range, 10-15 ms; FOV range, $24-25 \mathrm{~cm}$; matrix range, $192 \times 256$ to $320 \times 320$; section thickness, $1-5 \mathrm{~mm}$; intersection spacing, $1 \mathrm{~mm}$.

Midbrain, pons, and CC surfaces were measured at the midsagittal plane. Lines parallel to the mammillary-posterior commissural plane at the rostral and caudal pontine border were used to determine the midbrain and pons surfaces (Fig 1), according to the method of Kato et al. ${ }^{15}$ Middle cerebellar peduncle width was measured parasagittally, and superior cerebellar peduncle width was measured coronally (On-line Fig 1), according to the method of Longoni et al. ${ }^{16}$ The validity of the method has already been 
Table 2: ROC curve analysis of the discriminative power of morphometric measurements in patients with PSP, MSA, and CBD compared with all other groups

\begin{tabular}{lcccccc}
\hline & AUC (SD) & $\begin{array}{c}\boldsymbol{P} \\
\text { Value }\end{array}$ & $\begin{array}{c}\text { Cutoff } \\
\text { Value }\end{array}$ & $\begin{array}{c}\text { Sensitivity } \\
(\%)\end{array}$ & $\begin{array}{c}\text { Specificity } \\
(\%)\end{array}$ & $\begin{array}{c}\text { Likelihood } \\
\text { Ratio }\end{array}$ \\
\hline $\begin{array}{l}\text { PSP vs all groups } \\
\text { Midbrain }\end{array}$ & $0.94(0.03)$ & $<.001$ & $\leq 107$ & 79 & 97 & 24.54 \\
$\quad$ Midbrain/pons & $0.93(0.03)$ & $<.001$ & $\leq 0.22$ & 88 & 84 & 5.42 \\
$\quad$ Midbrain/CC & $0.92(0.04)$ & $<.001$ & $\leq 0.21$ & 92 & 80 & 4.58 \\
$\quad$ MRPI & $0.98(0.01)$ & $<.001$ & $>12.6$ & 91 & 95 & 18.48 \\
MSA (total) vs all groups & & & & & & \\
$\quad$ Pons & $0.74(0.08)$ & .001 & $\leq 475$ & 63 & 85 & 4.23 \\
$\quad$ Midbrain/pons & $0.86(0.05)$ & $<.001$ & $\geq 0.29$ & 74 & 90 & 7.05 \\
$\quad$ MRPI & $0.91(0.04)$ & $<.001$ & $\leq 7.32$ & 74 & 94 & 11.78 \\
CBD vs all groups & & & & & & \\
$\quad$ Corpus callosum & $0.79(0.09)$ & .004 & $\leq 470$ & 67 & 95 & 12.33 \\
$\quad$ CC P-A grad & $0.83(0.10)$ & .002 & $\leq 191$ & 75 & 97 & 25.12 \\
$\quad$ Pons/CC P-A grad & $0.81(0.08)$ & .003 & $\geq 2.22$ & 89 & 71 & 2.98 \\
\hline
\end{tabular}

Note:-AUC indicates area under the curve.

established as excellent, with low intra- and interrater variability of the measurements. ${ }^{16-18}$ All surfaces were manually traced by G.V. with the DICOM viewer R3.0-Sp3 (Philips Healthcare, Best, the Netherlands).

CC subsections were determined according to the classification of Hofer and Frahm. ${ }^{19}$ According to this classification, the $\mathrm{CC}$ can be subdivided into 5 sections $\left(\mathrm{CC}_{1}\right.$ to $\mathrm{CC}_{5}$ ), which represent, from anterior to posterior, the following brain regions: 1) prefrontal cortex, 2) premotor and supplementary motor cortex, 3) primary motor cortex, 4) primary sensory cortex, and 5) parietal, occipital, and temporal cortices (On-line Fig 2). To optimize diagnostic accuracy, we calculated and compared various indices based on CC subsections. Of these indices, the CC posteroanterior gradient $\left(\mathrm{CC}_{\mathrm{P}-\mathrm{A} \text { grad }}\right)$ provided the greatest discriminative power and was therefore used. The $\mathrm{CC}_{\mathrm{P}-\mathrm{A} \text { grad }}$ was calculated by subtracting the $\mathrm{CC}_{1}$ subsection from the remaining $\mathrm{CC}$ subsections $\left(\mathrm{CC}_{\mathrm{P}-\mathrm{A} \text { grad }}=\mathrm{CC}_{2}+\mathrm{CC}_{3}+\mathrm{CC}_{4}+\mathrm{CC}_{5}-\mathrm{CC}_{1}\right)$. The rationale behind the implementation of the $\mathrm{CC}_{\mathrm{P}-\mathrm{A} \text { grad }}$ was the relatively selective $\left(\mathrm{CC}_{2}\right.$ to $\left.\mathrm{CC}_{4}\right)$ atrophy in $\mathrm{CBD}$, with a preserved $\mathrm{CC}_{1}$ surface (On-line Fig 3).

The Fazekas periventricular and deep white matter hyperintensity score was used to measure while matter lesion burden. ${ }^{20}$

\section{Statistical Analysis}

Numeric variables were checked for normality and homogeneity of variances by the Shapiro-Wilk and Levene tests, respectively. Analysis of covariance, using the diagnosis, sex, and magnetic field strength ( $1.5 \mathrm{~T}$ versus $3 \mathrm{~T}$ ) as co-factors and age as a covariate, followed by post hoc Bonferroni correction for multiple comparisons or the Kruskal-Wallis test (followed by the Dunn post hoc test), was used as appropriate. Receiver operating characteristic curve analysis was applied for determination of the diagnostic value of each biomarker.

Two sets of analyses were performed. Initially, each diagnostic group (PSP, MSA, and CBD) was compared with all other groups, to examine the utility of planimetry MR imaging markers in the clinical scenario of a patient with parkinsonism of unknown etiology. The second analysis included pair-wise comparison of diagnoses that can be difficult to differentiate in clinical practice (ie, PSP versus multiple system atrophy with parkinsonism [MSA-P] versus $\mathrm{CBD}$ versus $\mathrm{PD})$.
Analyses were performed by SPSS Statistics, Version 22.0.0.0 (IBM, Armonk, New York; 2013) and GraphPad Prism, Version 5.03 (GraphPad Software, San Diego, California; 2009).

\section{RESULTS}

Clinical and Demographic Data

Groups did not differ among each other with respect to age and sex (Table 1). As expected, disease duration was significantly greater in those with $\mathrm{PD}$ compared with patients with Parkinson-plus. ANCOVA revealed significant effects by diagnostic group, but none of the cofactors and covariates affected the models significantly.

\section{Planimetry Measurements}

Patients with PSP exhibited significantly smaller midbrain surfaces compared with all other groups, resulting in smaller midbrain/CC and midbrain/pons ratios, as well as greater MRPI values (On-line Table). Furthermore, they presented with mild pons atrophy (numerically greater than MSA-P but lower than multiple system atrophy cerebellar type [MSA-C]).

Patients with MSA had smaller pons surfaces and pons-derived surface ratios as well as MRPI values. This difference came from patients with MSA-C because patients with MSA-P exhibited pons surfaces comparable with those of controls. Likewise, their midbrain and CC surfaces were like those of controls.

Patients with CBD had smaller CC surfaces compared with the other groups and numerically decreased midbrain surfaces (Online Fig 4).

\section{Comparison of Each Group versus All Other Groups}

The MRPI provided excellent discriminative power for PSP versus all other groups, with a value of $\geq 12.6$ providing $91 \%$ sensitivity and 95\% specificity (Table 2 and On-line Fig 5). The MRPI was better compared with other indices for the differential diagnosis of MSA versus all other groups, with moderate sensitivity (74\%) and excellent specificity (94\%) for a cutoff point of $\leq 7.32$.

The $\mathrm{CC}_{\mathrm{P}-\mathrm{A} \text { grad }}$ with a cutoff point of $\leq 191 \mathrm{~mm}^{2}$ provided $97 \%$ specificity and $75 \%$ sensitivity for the diagnosis of CBD. A CC surface of $\leq 470 \mathrm{~mm}^{2}$ provided moderate sensitivity $(67 \%)$ and excellent specificity (95\%) for the diagnosis of CBD.

\section{Pair-Wise Group Comparisons}

The MRPI achieved very good discrimination between PSP and MSA-P with $100 \%$ specificity and sensitivity (Table 3). Likewise, the MRPI discriminated patients with PSP from those with PD with a sensitivity of $86 \%$ and a specificity of $100 \%$. The midbrain/CC $\mathrm{CC}_{\mathrm{P}-\mathrm{A} \text { grad }}$ ratio provided $86 \%$ sensitivity and $88 \%$ specificity in the differential diagnosis of PSP from CBD. No MR imaging measurement was clinically useful in differentiating patients with PD from those with MSA-P. The $\mathrm{CC}_{\mathrm{P}-\mathrm{A} \text { grad }}$ provided moderate sensitivity and excellent specificity in the differential diagnosis of patients with CBD from those with PD (75\% and 100\%, respectively). Midbrain surface discriminated between patients with MSA-P and CBD with a sensitivity and specificity of $89 \%$. 
Table 3: Pair-wise analysis of the discriminative power of morphometric measurements with ROC curve analysis

\begin{tabular}{|c|c|c|c|c|c|c|c|c|c|}
\hline & MRPI & $\begin{array}{c}\text { Midbrain } \\
\left(\mathrm{mm}^{2}\right)\end{array}$ & $\begin{array}{l}\text { Pons } \\
\left(\mathrm{mm}^{2}\right)\end{array}$ & $\mathrm{CC}\left(\mathrm{mm}^{2}\right)$ & $\begin{array}{c}C_{\text {P-A grad }} \\
\left(\mathrm{mm}^{2}\right)\end{array}$ & $\begin{array}{l}\text { Midbrain/ } \\
\text { Pons }\end{array}$ & Midbrain/CC & Midbrain/CC $C_{P-A}$ grad & Pons/CC \\
\hline \multicolumn{10}{|c|}{ PSP vs MSA-P } \\
\hline Cutoff & $\geq 11.0$ & $\leq 129$ & NS & NS & NS & $\leq 0.22$ & $\leq 0.19$ & $\leq 0.45$ & NS \\
\hline Sens (\%) & 100 & 96 & & & & 88 & 75 & 86 & \\
\hline Spec (\%) & 100 & 89 & & & & 89 & 100 & 100 & \\
\hline \multicolumn{10}{|l|}{ PSP vs PD } \\
\hline Cutoff & $\geq 12.7$ & $\leq 109$ & $\leq 523$ & NS & NS & $\leq 0.21$ & $\leq 0.19$ & $\leq 0.40$ & NS \\
\hline Sens (\%) & 86 & 79 & 75 & & & 71 & 75 & 77 & \\
\hline Spec (\%) & 100 & 94 & 78 & & & 100 & 100 & 94 & \\
\hline \multicolumn{10}{|l|}{ PSP vs CBD } \\
\hline Cutoff & $\geq 13.7$ & $\leq 100$ & NS & $\geq 470$ & $\geq 192$ & $\leq 0.19$ & $\leq 0.18$ & $\leq 0.45$ & $\geq 0.87$ \\
\hline Sens (\%) & 73 & 71 & & 92 & 91 & 67 & 71 & 86 & 46 \\
\hline Spec(\%) & 100 & 100 & & 67 & 75 & 89 & 100 & 88 & 100 \\
\hline \multicolumn{10}{|c|}{ MSA-P vs PD } \\
\hline Cutoff & $\leq 8.0$ & NS & NS & NS & NS & NS & NS & NS & NS \\
\hline Sens (\%) & 56 & & & & & & & & \\
\hline Spec (\%) & 89 & & & & & & & & \\
\hline \multicolumn{10}{|l|}{ CBD vs PD } \\
\hline Cutoff & NS & $\leq 146$ & $\leq 563$ & $\leq 479$ & $\leq 193$ & NS & NS & NS & NS \\
\hline Sens (\%) & & 100 & 100 & 67 & 75 & & & & \\
\hline Spec (\%) & & 67 & 56 & 94 & 100 & & & & \\
\hline \multicolumn{10}{|c|}{ MSA-P vs CBD } \\
\hline Cutoff & $\leq 11.0$ & $\geq 129$ & NS & $\geq 478$ & $\geq 199$ & NS & NS & NS & NS \\
\hline Sens (\%) & 100 & 89 & & 89 & 100 & & & & \\
\hline Spec (\%) & 63 & 89 & & 67 & 75 & & & & \\
\hline
\end{tabular}

Note:-NS indicates not significant; Sens, sensitivity; Spec, specificity; ROC, receiver operating characteristic.

\section{DISCUSSION}

The present study aimed at examining the utility of MR imaging planimetry measurements as surrogate markers of midbrain, pons, and CC atrophy, in the differential diagnosis of patients with Parkinson-plus syndrome.

Patients with PSP in our cohort were characterized by severe midbrain and, to a lesser extent, pons atrophy. Of the patients with MSA, only those with MSA-C presented with pons atrophy. On the contrary, patients with MSA-P were not characterized by pons atrophy. Patients with CBD had predominantly CC atrophy and mild midbrain atrophy. Patients with PD did not differ from control subjects.

Most studies support midbrain and superior cerebellar peduncle atrophy as predominant features in patients with PSP. ${ }^{21,22}$ This translates into a significantly higher MRPI and lower midbrain-to-pons surface ratio values in patients with PSP. ${ }^{10,23,24}$ The MRPI was indeed the most potent imaging marker for the differential diagnosis of PSP from all other groups in our cohort. This also applied to the differential diagnosis of PSP from MSA-P or PD. It has been reported that these imaging findings are present early in the disease course of patients with PSP, often before the complete clinical phenotype of Richardson syndrome is evident. $^{21,22,25}$ This finding renders the MRPI and midbrain-topons surface ratio useful in the early differentiation of patients with PSP from those with MSA-P and PD.

Low MRPI values have been suggested to further aid in the differential diagnosis of MSA from other causes of parkinsonism. ${ }^{17,26-28}$ In our cohort, low MRPI values were moderately sensitive (74\%) and highly specific (94\%) in the differentiation of patients with MSA from all other groups. This however was due to the inclusion of patients with MSA-C in the MSA group. These patients have particularly low MRPI values, due to pronounced pons and middle cerebellar peduncle atrophy. After we excluded patients with MSA-C, the MRPI was not clinically useful in the differential diagnosis of patients with MSA-P from those with PD or CBD. Thus, we could not establish the utility of the MRPI in the discrimination of patients with MSA-P from other patients with parkinsonism, except for PSP. Considering that patients with MSA-C are rarely confused with other patients with Parkinsonplus, due to their predominant cerebellar symptoms, low MRPI values may not provide clinically relevant assistance in most cases.

Patients with CBD in our cohort had decreased CC surfaces compared with other groups. A low $\left(<470 \mathrm{~mm}^{2}\right)$ corpus callosum surface was highly specific (95\%) for CBD but lacked sensitivity $(67 \%)$. This finding is in agreement with a single planimetry study that demonstrated CC atrophy in patients with CBD compared with PSP, with substantial between-group overlap however. ${ }^{29} \mathrm{CC}$ subsection analysis indicated a posteroanterior CC atrophy gradient in $\mathrm{CBD}$, with relative preservation of the $\mathrm{CC} 1$ segment (prefrontal cortex). PSP, on the other hand, had more pronounced anterior CC atrophy. This generated the $\mathrm{CC}_{\mathrm{P}-\mathrm{A} \text { grad }}$, which provided improved sensitivity (75\%) and specificity (97\%) in the diagnosis of $\mathrm{CBD}$ versus all other groups. The $\mathrm{CC}_{\mathrm{P}-\mathrm{A} \text { grad }}$ was superior to all other indices in the differentiation of CBD from $\mathrm{PD}$, with excellent specificity (100\%) and moderate sensitivity (75\%). Furthermore, the midbrain/ $\mathrm{CC}_{\mathrm{P}-\mathrm{A} \text { grad }}$ ratio was superior to the MRPI in the differential diagnosis of PSP from CBD (sensitivity $86 \%$, specificity $88 \%$ ).

Most planimetry studies of Parkinson-plus syndromes in the field lack pathologic confirmation, as is the case with our study. To compensate for the problem, we exclusively included prospectively diagnosed patients who fulfilled the "probable" diagnostic criteria. To keep the possibility of misdiagnosis as low as possible, all patients were followed up for at least 2 years. None of the patients presented with any atypical clinical features during this 
period. Furthermore, we used CSF biochemical profile analysis to exclude patients with an underlying Alzheimer disease pathology.

The sample size of our cohort is moderate, as is expected for diseases as rare as Parkinson-plus syndromes, but comparable with most studies on the subject. Intra- and interrater agreement of MR imaging surface measurements was not tested in the present study because it has already been proved excellent in previous studies. ${ }^{17,22,25,28}$

Further studies of larger cohorts of patients with Parkinsonplus syndrome, incorporating clinical, imaging, and pathologic data, would assist in further elucidating the complex interaction among underlying pathology, atrophy profile, and clinical phenotype. These studies could examine the utility of more focused planimetry markers, which take into account the topographic selectivity of atrophy (such as the $\mathrm{CC}_{\mathrm{P}-\mathrm{A} \text { grad }}$ ).

\section{CONCLUSIONS}

MR imaging planimetry can facilitate the differential diagnosis of patients with parkinsonism. Midbrain surface and relevant indices (such as the MRPI) are already established markers of PSP. Likewise, corpus callosum surface indices are promising markers of CBD. MR imaging planimetry, however, does not assist in the differential diagnosis of MSA-P from PD.

\section{REFERENCES}

1. Rolland Y, Vérin M, Payan C, et al; NNIPPS Study Group. A new MRI rating scale for progressive supranuclear palsy and multiple system atrophy: validity and reliability. J Neurol Neurosurg Psychiatry 2011; 82:1025-32 CrossRef Medline

2. Respondek G, Roeber S, Kretzschmar H, et al. Accuracy of the National Institute for Neurological Disorders and Stroke/Society for Progressive Supranuclear Palsy and neuroprotection and natural history in Parkinson plus syndromes criteria for the diagnosis of progressive supranuclear palsy. Mov Disord 2013;28:504-09 CrossRef Medline

3. Osaki Y, Ben-Shlomo Y, Lees AJ, et al. A validation exercise on the new consensus criteria for multiple system atrophy. Mov Disord 2009;24:2272-76 CrossRef Medline

4. Armstrong MJ, Litvan I, Lang AE, et al. Criteria for the diagnosis of corticobasal degeneration. Neurology 2013;80:496-503 CrossRef Medline

5. Hauw JJ, Daniel SE, Dickson D, et al. Preliminary NINDS neuropathologic criteria for Steele-Richardson-Olszewski syndrome (progressive supranuclear palsy). Neurology 1994;44:2015-19 CrossRef Medline

6. Yamauchi H, Fukuyama H, Nagahama Y, et al. Atrophy of the corpus callosum, cognitive impairment, and cortical hypometabolism in progressive supranuclear palsy. Ann Neurol 1997;41:606-14 CrossRef Medline

7. Wenning GK, Tison F, Ben Shlomo Y, et al. Multiple system atrophy: a review of 203 pathologically proven cases. Mov Disord 1997;12: 133-47 CrossRef Medline

8. Dickson DW, Bergeron C, Chin SS, et al; Office of Rare Diseases of the National Institutes of Health. Office of Rare Diseases neuropathologic criteria for corticobasal degeneration. J Neuropathol Exp Neurol 2002;61:935-46 CrossRef Medline

9. Yamauchi H, Fukuyama H, Nagahama Y, et al. Atrophy of the corpus callosum, cortical hypometabolism, and cognitive impairment in corticobasal degeneration. Arch Neurol 1998;55:609-14 CrossRef Medline

10. Quattrone A, Nicoletti G, Messina D, et al. MR imaging index for differentiation of progressive supranuclear palsy from Parkinson disease and the Parkinson variant of multiple system atrophy. $R a$ diology 2008;246:214-21 CrossRef Medline
11. Litvan I, Agid Y, Calne D, et al. Clinical research criteria for the diagnosis of progressive supranuclear palsy (Steele-RichardsonOlszewski syndrome): report of the NINDS-SPSP international workshop. Neurology 1996;47:1-9 CrossRef Medline

12. Gilman S, Wenning GK, Low PA, et al. Second consensus statement on the diagnosis of multiple system atrophy. Neurology 2008;71: 670-76 CrossRef Medline

13. Paraskevas GP, Kasselimis D, Kourtidou E, et al. Cerebrospinal fluid biomarkers as a diagnostic tool of the underlying pathology of primary progressive aphasia. J Alzheimers Dis 2017;55:1453-61 CrossRef Medline

14. Hughes AJ, Daniel SE, Kilford L, et al. Accuracy of clinical diagnosis of idiopathic Parkinson's disease: a clinico-pathological study of 100 cases. J Neurol Neurosurg Psychiatry 1992;55:181-84 CrossRef Medline

15. Kato N, Arai K, Hattori T. Study of the rostral midbrain atrophy in progressive supranuclear palsy. J Neurol Sci 2003;210:57-60 CrossRef Medline

16. Longoni G, Agosta F, Kostić VS, et al. MRI measurements of brainstem structures in patients with Richardson's syndrome, progressive supranuclear palsy-parkinsonism, and Parkinson's disease. Mov Disord 2011;26:247-55 CrossRef Medline

17. Cosottini M, Ceravolo R, Faggioni L, et al. Assessment of midbrain atrophy in patients with progressive supranuclear palsy with routine magnetic resonance imaging. Acta Neurol Scand 2007;116: 37-42 CrossRef Medline

18. Morelli M, Arabia G, Salsone M, et al. Accuracy of magnetic resonance parkinsonism index for differentiation of progressive supranuclear palsy from probable or possible Parkinson disease. Mov Disord 2011;26:527-33 CrossRef Medline

19. Hofer S, Frahm J. Topography of the human corpus callosum revisited; comprehensive fiber tractography using diffusion tensor magnetic resonance imaging. Neuroimage 2006;32:989-94 CrossRef Medline

20. Fazekas F, Chawluk JB, Alavi A. MR signal abnormalities at $1.5 \mathrm{~T}$ in Alzheimer's dementia and normal aging. AJR Am J Roentgenol 1987; 8:421-26 CrossRef Medline

21. Aiba I, Hashizume Y, Yoshida M, et al. Relationship between brainstem MRI and pathological findings in progressive supranuclear palsy: study in autopsy cases. J Neurol Sci 1997;152:210-17 CrossRef Medline

22. Slowinski J, Imamura A, Uitti RJ, et al. MR imaging of brainstem atrophy in progressive supranuclear palsy. J Neurol 2008;255:37-44 CrossRef Medline

23. Hussl A, Mahlknecht P, Scherfler C. Diagnostic accuracy of the magnetic resonance Parkinsonism index and the midbrain-to-pontine area ratio to differentiate progressive supranuclear palsy from Parkinson's disease and the Parkinson variant of multiple system atrophy. Mov Disord 2010;25:2444-49 CrossRef Medline

24. Kim YH, Ma HI, Kim YJ. Utility of the midbrain tegmentum diameter in the differential diagnosis of progressive supranuclear palsy from idiopathic Parkinson's disease. J Clin Neurol 2015;11:268-74 CrossRef Medline

25. Morelli M, Arabia G, Novellino F, et al. MRI measurements predict PSP in unclassifiable parkinsonisms: a cohort study. Neurology 2011;77:1042-47 CrossRef Medline

26. Massey LA, Jäger HR, Paviour DC, et al. The midbrain to pons ratio: a simple and specific MRI sign of progressive supranuclear palsy. Neurology 2013;80:1856-61 CrossRef Medline

27. Kaasinen V, Kangassalo N, Gardberg M, et al. Midbrain-to-pons ratio in autopsy-confirmed progressive supranuclear palsy: replication in an independent cohort. Neurol Sci 2015;36:1251-53 CrossRef Medline

28. Oba $H$, Yagishita $A$, Terada $H$, et al. New and reliable MRI diagnosis for progressive supranuclear palsy. Neurology 2005;64:2050-55 CrossRef Medline

29. Gröschel K, Hauser T-K, Luft A, et al. Magnetic resonance imaging-based volumetry differentiates progressive supranuclear palsy from corticobasal degeneration. Neuroimage 2004;21:714-24 CrossRef Medline 